\title{
Percepción del Clima Motivacional de Clase en Estudiantes Adultos no Universitarios
}

\section{Perception of Classroom Motivational Climate in Adult Non- University Students}

\author{
Jesús Alonso Tapia y $\mathbf{M}^{\mathrm{a}}$ Adelaida Moral Bosch \\ Universidad Autónoma de Madrid-España
}

\begin{abstract}
Resumen. El presente estudio tiene dos objetivos: a) Comprobar en qué medida el Clima Motivacional de Clase (CMS) constituido por las pautas de actuación del profesor tiene las mismas características e implicaciones para los adultos no universitarios que las que se han encontrado en adolescentes utilizando el Cuestionario de Clima Motivacional de Clase (CMCQ) (Alonso Tapia y Fernández (2008); y b) comprobar en qué medida modulan la percepción del clima de clase: 1) las características motivacionales con que los alumnos acceden a clase y 2) las características de los profesores que las imparten y condicionan sus pautas de actuación. Los resultados obtenidos tras examinar con el CMCQ y otros cuestionarios a 185 estudiantes adultos no universitarios y realizar análisis confirmatorios, multigrupo, de correlaciones y de regresión han puesto de manifiesto las semejanzas y diferencias en la percepción del CMC entre ambos colectivos, en las consecuencias de la misma y en los factores que la afectan, resultados con importantes implicaciones teóricas y prácticas que se comentan en el artículo.

Palabras clave: clima motivacional de clase, educación de adultos, metas de los alumnos, teoría de metas, calidad del entorno de aprendizaje, intervención educativa.
\end{abstract}

\begin{abstract}
This paper has two objectives. Firstly, to know if the Classroom Motivational Climate questionnaire (CMC), developed by Alonso Tapia and Fernández (2008), has the same characteristics and implications in adult non-university students as in secondary/high school students. Secondly, we aimed to find out to what extent the following factors affect perception of class climate: a) the motivational characteristics of the students, and b) the motivational characteristics of their teachers. Results obtained after giving 185 adult nonuniversity students the questionnaires, and carrying out confirmatory factor analysis, multigroup analysis, and correlation and regression analysis, showed similarities and differences in CMC perception between the two groups, in its consequences, and in the factors on which it depends. These results have important theoretical and practical implications which are commented on in the study.

Key words: classroom motivational climate, adult education, classroom goal structure, students' goals, goal theory, learning environment quality, educational intervention.
\end{abstract}

Durante el curso académico que la segunda autora estuvo como orientadora en un centro educativo de adultos no universitarios, ésta tomó conciencia

La correspondencia de este artículo dirigirla a: Jesús Alonso-Tapia. Facultad de Psicología. Universidad Autónoma. c/ Iván Pavlov, 6. Madrid.E-mail: jesus.alonso@uam.es del hecho de que los profesores consultados atribuían la falta de rendimiento de sus alumnos a causas totalmente ajenas a factores motivacionales. Las explicaciones iban desde la falta de capacidad cognitiva, hasta el desconocimiento de estrategias y técnicas para estudiar o simplemente la presencia de 
hechos externos muy estresantes que "obligaban" a los estudiantes a abandonar. La motivación se interpretaba como un suceso externo aleatorio que podía darse o no de manera espontánea dentro de una clase, pero que siempre escapaba al control del profesor. Al tener la creencia de que era un factor incontrolable, los profesores consultados no invertían el tiempo y el esfuerzo suficiente como para crear un clima motivacional que pudiera ser beneficioso para sus alumnos. Estos hechos, motivaron la realización de este trabajo, que aparece con la idea de conocer más sobre la motivación de los estudiantes adultos no universitarios y de cómo se relaciona esta motivación con el rendimiento académico, así como de poder ofrecer a los profesores sugerencias sobre cómo producir mejoras en los procesos motivacionales de sus estudiantes y de sí mismos, como paso previo para solucionar muchos de los problemas académicos de esta población.

La cuestión sobre qué hacer para motivar se ha investigado de forma extensa tanto en el contexto de la Enseñanza Primaria, como de la Enseñanza Secundaria y de los estudios universitarios. Sin embargo, ¿qué ocurre con los estudiantes adultos no universitarios? Es notable la inexistencia de estudios significativos en este ámbito. Tal y como plantea el problema la mayoría de la investigación consultada, la clave está en que, dentro de una clase, siempre se crea un contexto de trabajo definido por las pautas de actuación de los profesores, que se llama clima motivacional de aula. Este clima, se supone, por un lado, que está influido por las características motivacionales de los profesores, esto es, por sus metas y sus expectativas docentes en cuanto a la posibilidad de motivar, por su optimismo o su pesimismo generalizado, y por su actitud ante el uso de la competición (Alonso Tapia, 1992a). Y, por otro lado, se supone que influye en distintos aspectos de la motivación de los alumnos -metas, intereses, habilidad percibida y esfuerzo con que se enfrentan a la tarea académica (Alonso Tapia y Fernández Heredia, 2008, 2009; Renninger, Hidi \& Krapp, 1992; Bandura, 1997). Además, de forma indirecta o a través de tales aspectos parece tener consecuencias tanto en su rendimien- to académico como en la satisfacción que reportan con respecto al profesor que les enseña la asignatura.

De esta manera, parece ser que el docente no solo no es un simple observador de los hechos motivacionales que ocurren dentro de su clase, sino que, no puede no influir en la motivación de sus estudiantes.

Las suposiciones anteriores sugirieron la conveniencia de estudiar los tres problemas siguientes en los sujetos adultos no universitarios:

a) Primero, si las características que configuran el clima motivacional de clase (CMC) son las mismas que para otros niveles académicos. Para ello, nos hemos basado en los estudios sobre el CMC de Alonso Tapia y Fernández (2008, 2009) realizados con adolescentes, debido a los excelentes resultados psicométricos que se han obtenido.

b) Segundo, averiguar cuál es el grado en que las características motivacionales de profesores y alumnos afectan a la percepción del CMC por parte de los segundos. Nos hemos apoyado tanto en los supuestos sobre la relación entre características motivacionales de los docentes y CMC descritos por Alonso Tapia (1992a) como en nuestros propios supuestos sobre la relación entre la orientación motivacional de los estudiantes y el modo en que puede afectarles en su percepción del CMC.

c) Tercero, identificar cuál es el grado en que el CMC afecta las características motivacionales de los estudiantes sensibles al contexto -intereses, habilidad percibida, expectativas de éxito $y$ esfuerzo por aprender de los alumnos en relación con la tarea-, objetivo para el que nos hemos basado en los trabajos de Alonso Tapia y Fernández (2008, 2009).

Partiendo de las suposiciones anteriores, las preguntas que nos formulamos fueron las siguientes:

1. ¿Cuáles son las variables que definen el CMC en la población de adultos no universitarios? ¿Son las mismas que en los adolescentes?

2. ¿Cuáles son los factores personales de los alumnos adultos que modulan la percepción del CMC? 
3. ¿Cuáles son los factores personales de los profesores que modulan la percepción del CMC por parte de los alumnos?

4. ¿Las diferencias entre los perfiles motivacionales de los profesores de adultos son lo suficientemente significativas como para obtener diferencias significativas en los diferentes CMC que generan?

5. ¿Qué efecto tiene el CMC percibido en el interés, la habilidad percibida, el esfuerzo y las expectativas de resultado $\mathrm{y}$, a través de éstas, en la satisfacción y el rendimiento?

\section{Marco teórico}

¿Qué supuestos han servido de base para orientar la realización de este trabajo? Son de distinto tipo, por lo que se presentan organizados en torno a distintos enunciados.

1) Los profesores tienen diferentes patrones de enseñanza que podrían influir en la configuración del CMC de un aula.

Los estudios consultados, sugieren que las variables motivacionales de los profesores que pueden influir en el patrón de enseñanza que luego desarrollan en la clase son las siguientes: a) actitud de rechazo de trabajo en grupo: el profesor prefiere el uso de estrategias que se centran en valorarse a sí mismo como docente a través de los resultados de sus estudiantes y por tanto no tiene mucha confianza en ser capaz de motivar; b) optimismo motivacional: el profesor tiene expectativas altas de auto-eficacia e invertirá más esfuerzo en motivar a sus alumnos; c) actitud favorable al uso de la competición: el docente cree en el uso de la comparación o de la amenaza que supone el suspenso como elementos motivadores; y por último, d) pesimismo generalizado: el profesor supone que los factores externos a la actividad de aprendizaje tienen un papel muy determinante en la motivación, así que piensa que apenas los puede controlar o compensar. (Alonso Tapia, 1992a).

Por su parte, el concepto de "clima motivacional de aula" se refiere al conjunto de patrones de actua- ción del profesorado que configuran el entorno de aprendizaje de los alumnos (Ames, 1992). Se creó con la intención de relacionar las metas de logro de los alumnos con los factores ambientales de una clase. La mayoría de los estudios consultados, indican que los patrones de enseñanza que configuran el CMC pueden facilitar o dificultar el predominio de las metas de aprendizaje entre los estudiantes. En concreto, el trabajo de investigación de Alonso Tapia y Fernández Heredia se basa en esta premisa y construye su instrumento de evaluación CMC-Q, a partir de una síntesis de las siguientes estrategias de enseñanza facilitadoras de un CMC de aprendizaje recogidas por Alonso Tapia y Pardo (2006) y Urdan y Turner (2005):

$\left.1^{\circ}\right)$ Al inicio de las actividades de aprendizaje, hay que activar la intención de aprender. Para ello, se deberían usar estrategias de presentación de información nueva o sorprendente o usar tareas que muestren la utilidad de lo que se va a enseñar (Alonso Tapia, 2005 a y b; Keller, 1985).

$\left.2^{\circ}\right)$ Durante las actividades de aprendizaje, es necesario mantener la atención de los alumnos centrada en el proceso más que en el producto de aprendizaje. Para ello, sería conveniente: a) cuando se introducen nuevos contenidos o actividades, procurar focalizar la atención estudiantil en el proceso de aprendizaje y en metas intrínsecas, en lugar de en los resultados, la comparación social o la evaluación (Urdan y Turner, 2005). También deberían elaborar un plan de actividades que le ayuden a seguir las explicaciones y a auto-regularse; b) cuando se da la información y las explicaciones, el profesor debe asegurarse que sus alumnos están teniendo una experiencia de comprensión y competencia personales en su asignatura (Assor y Kaplan, 2001). Para ello, hará uso de un discurso jerárquico y coherente, realizará una inducción a la participación y el uso de ilustraciones y ejemplos para que los estudiantes puedan construir representaciones mentales más concretas de las ideas abstractas (Alonso Tapia y López, 1999); c) en la interacción con sus alumnos, el profesor, debería: permitirles participar de forma espontánea, escucharles con atención, pedir más 
explicación a las respuestas dadas si fuera necesario, reforzarles al participar, señalar los elementos o respuestas positivas aunque estén incompletas, preguntar por las razones que están detrás de las respuestas incorrectas, y tener tiempo para atender a los estudiantes que pidan ayuda o evitar la comparación entre alumnos, para favorecer la percepción de igualdad; d) y, por último, cuando es necesario proponer actividades de aprendizaje que impliquen independencia en el estudiante, el profesor debe: sugerir el establecimiento de metas personales, ofrecer opciones, enseñar a sus alumnos a preguntarse “¿cómo puedo hacerlo?”, sugerir la división de tareas en pasos más pequeños, quitarle importancia a la petición de ayuda, dando feedback y ayuda tanto como sea necesario, subrayando el progreso individual y el papel activo del estudiante en el mismo y procurando ajustar el ritmo de trabajo (Pardo y Alonso Tapia, 1990).

$\left.3^{\circ}\right)$ Al finalizar las actividades de aprendizaje, o durante las mismas, siempre y cuando haya que hacer una valoración del trabajo realizado. La evaluación puede influir de manera positiva en la motivación si es una evaluación para el aprendizaje, y por tanto, cumple con las condiciones siguientes: a) ofrece información al estudiante o profesor que puede ayudar al estudiante a resolver dificultades y a autorregular su aprendizaje; b) usa tareas que implican novedad y explicitan el tipo de metas a lograr, así como los factores de los estudiantes que favorecen el cambio conceptual y procedimental, y si dan ayuda específica, está basada en la evaluación; c) y por último, si los profesores evitan mensajes y prácticas de aula que señalen la relevancia de la evaluación para objetivos extrínsecos a la propia comprensión de los contenidos. (Self-Brown y Mathews-II, 2003).

2) Cada alumno tiene unas características motivacionales diferentes y es posible que éstas condicionen su percepción del CMC.

Se llaman metas de logro a los objetivos personales que suele buscar preferentemente un estudiante con su trabajo académico, objetivos que pueden ser de dominio (mastery), de desempeño (performance) o de evitación (avoidance) (Alonso Tapia, Huertas y Ruiz, 2010).

Si la meta de logro es de dominio, el alumno tiene orientación a aprender y disfruta cuando lo consigue. Si la meta de logro es de desempeño (resultado), el estudiante busca más bien un lucimiento personal -a menudo concretada en la calificación- si el desempeño está asociado a la ejecución, o busca la evitación de la experiencia emocional negativa asociada al hecho real de fracaso, si el desempeño está asociado a la evitación (Covington, 2000; Alonso Tapia, 2005a). En ambos casos, el desempeño está asociado con el resultado en los aprendizajes a largo plazo, siendo preferibles las metas de "logro de aprendizaje".

La meta de logro de un estudiante, puede influir en su percepción del CMC y por lo tanto, en el predominio de un tipo de meta u otro dentro de esa asignatura en concreto. ¿Cómo interactúa la meta de logro del estudiante y el clima motivacional de clase? Es posible que una estructura motivacional de clase orientada a aprendizaje permita lograr buenos resultados no sólo de alumnos que buscan aprender, sino también de los que buscan otras metas (Brophy, 2005).. Por ejemplo, los alumnos cuya meta es el resultado (desempeño) se podrían adaptar a una situación de aprendizaje. Y también es posible que un estudiante con metas de dominio en un CMC altamente competitivo pueda tener dificultades motivacionales porque este clima chocará con sus metas (Alonso Tapia y Fernández Heredia, 2008; Bobbitt, 2007).

3) El CMC afecta las características motivacionales de los alumnos sensibles al contexto y que influyen en el rendimiento.

Se entiende por características motivacionales de los alumnos ligadas sensibles al contexto los intereses, la habilidad percibida, las expectativas de resultado y el esfuerzo con los que se enfrentan a las tareas académicas (Alonso Tapia, 2005b). Pues bien, la investigación indica que el CMC influye en estas características de los estudiantes del modo siguiente.

En primer lugar, parece ser que el interés que un tema o una asignatura dada despierta en un alumno 
no depende sólo de que éste tenga una predisposición relativamente permanente de acercamiento o evitación a una situación académica dada, sino que también es sensible a las características de la situación, es decir, depende de condiciones educativas externas, siendo susceptible de ser influido por estas. Las condiciones externas han de ser, pues, las adecuadas como para poder despertar el interés en el alumno (Hidi, 2006). ¿Qué condiciones educativas son las más adecuadas para fomentar interés? Según la segunda premisa teórica de la que hemos partido, es preciso al introducir una tarea utilizar estrategias que activen la curiosidad -plantear interrogantes, presentar hechos que rompan las expectativas del alumno, plantear desafíos razonables-, y facilitar la atención al proceso, la autoevaluación y la atención a la experiencia de progreso durante la tarea y al final de la misma (Alonso Tapia, 2005b, Urdan y Turner, 2005).

En segundo lugar, parece ser que la habilidad percibida para hacer frente a una tarea es sensible al $\mathrm{CMC}$, clima que parece influir tanto en las expectativas de autoeficacia como en las expectativas de resultado de los alumnos (Bandura, 1977, 1987, 1997). El concepto "expectativas de auto-eficacia" se refiere al grado en que el alumno cree que es capaz de hacer o no lo necesario para conseguir el objetivo de que una tarea, mientras que el concepto "expectativas de resultado" se refiere al éxito o fracaso que el estudiante anticipa. En muchos casos, el docente supone que ambos tipos de expectativas son difíciles de cambiar. Sin embargo, parece que un entorno que oriente a los alumnos hacia el dominio de la tarea puede ofrecer experiencias de éxito suficientes, como para influir positivamente en ambas (Bandura, 1987; Bobitt, 2007).

Finalmente, en tercer lugar, el CMC parece influir en el esfuerzo por aprender que el estudiante invierte en la asignatura dada y por tanto, en la meta que acaba adoptando en esa clase. Ahora bien, el esfuerzo en sí mismo contribuye a la experiencia de aprendizaje y de progreso y estará probablemente ligado a la satisfacción con la materia y con el profesor que la enseña. Así que, a la pregunta de ¿cuál es la rela- ción entre esfuerzo y satisfacción? Parece que la investigación indica la existencia de una relación bidireccional entre ambas variables que parece que se retroalimentan la una a la otra. Y ¿cómo se relaciona el CMC con el esfuerzo? La investigación consultada indica que un CMC de dominio, produce mucho más esfuerzo en los estudiantes que uno de desempeño (Alonso Tapia y Fernández Heredia, 2008, 2009).

4) El CMC orientado al aprendizaje afecta positivamente producir tanto a la satisfacción del estudiante con su profesor, como a su rendimiento académico.

Parece ser que cuando un profesor logra con sus patrones docentes configurar un CMC de aprendizaje, suele dar lugar a un clima de aula percibido por los alumnos como orientado al aprendizaje. Es un tipo de CMC que suele mejorar el grado de satisfacción del estudiante con su profesor, pero siempre y cuando se consiga que el estudiante adopte en la práctica una meta de aprendizaje en esa asignatura (Alonso Tapia y Fernández Heredia, 2008, 2009).

Además y en segundo lugar, cuando se adopta un CMC orientado al aprendizaje, se lleva a cabo el tipo de evaluación que ya hemos llamado en este resumen evaluación para el aprendizaje. Recordemos que a modo de estrategia de enseñanza facilitadora de un $\mathrm{CMC}$ de aprendizaje, el tipo de evaluación que puede producir una mejora en los resultados académicos, a de ser informativa, hacer explícitas las metas a seguir y estar centrada en la comprensión (Self-Brown y Mathews-II, 2003; Alonso Tapia y Pardo, 2006).

En función de estos supuestos teóricos, elaboramos las siguientes hipótesis que contrastamos en la parte empírica de nuestro trabajo:

a) Cabe caracterizar el Clima Motivacional de Clase del mismo modo en el caso de los alumnos adultos que en el de la población de estudiantes adultos no universitarios, esto es, los distintos componentes del clima de aprendizaje descritos por Alonso Tapia y Pardo (2006) y Alonso Tapia y Fernández Heredia (2008) tienden a ser percibidos en asociación. 
b) En la medida en que el CMC esté orientado al aprendizaje en una asignatura, cabe esperar que serán mayores el interés, la habilidad percibida, las expectativas de resultado y el esfuerzo por aprender invertido por los estudiantes en dicha asignatura.

c) En la medida en que promover las características motivacionales señaladas en el punto "b" puede generar emociones positivas en los alumnos, cabe esperar que el CMC influya de manera positiva en la satisfacción con el profesor.

d) En la medida en que movilizar las características motivacionales indicadas, produce una influencia del CMC en la satisfacción con el profesor, cabe esperar que también influya de manera positiva en el rendimiento académico.

e) Se espera que un profesor actúe de manera diferente en un aula según las metas que persiga con su práctica docente y que, en la medida en que estas metas se orienten al aprendizaje, influirán positivamente en la percepción del CMC en su aula.

f) Así mismo, cabe esperar que en la medida en que la orientación motivacional de los alumnos al acceder a clase se centre en el aprendizaje o al resultado, la percepción del CMC se verá afectada en función de la congruencia entre el CMC real creado por el profesor y la orientación motivacional.

A continuación se indica cómo se realizó el trabajo empírico de nuestra investigación.

\section{Trabajo empírico}

\section{Método}

\section{Muestra}

La muestra para este estudio está compuesta de 185 sujetos estudiantes adultos no universitarios, $52.4 \%$ mujeres y $47.6 \%$ hombres. Provenían de cuatro centros distintos: $62.7 \%$ del Centro de Adultos Polígono de la localidad de Toledo, 16.2\% de la Escuela de Adultos La Sisla de la localidad de Toledo, $10.3 \%$ del Centro de Adultos de la localidad de Illescas y $10.8 \%$ del Centro de Adultos Teresa Enríquez de la localidad de Torrijos. Estaban distribuidos en cuatro cursos de Educación Secundaria Obligatoria Para Adultos, (ESPA): $5.9 \%$ de $1^{\circ}$; $34.1 \%$ de $2^{\circ} ; 31.9 \%$ de $3^{\circ}$ y $28.1 \%$ de $4^{\circ}$.

En la Figura 1 puede verse que las edades de la muestra de estudiantes eran muy diversas, estando

Figura 1. Distribución de la muestra por edad

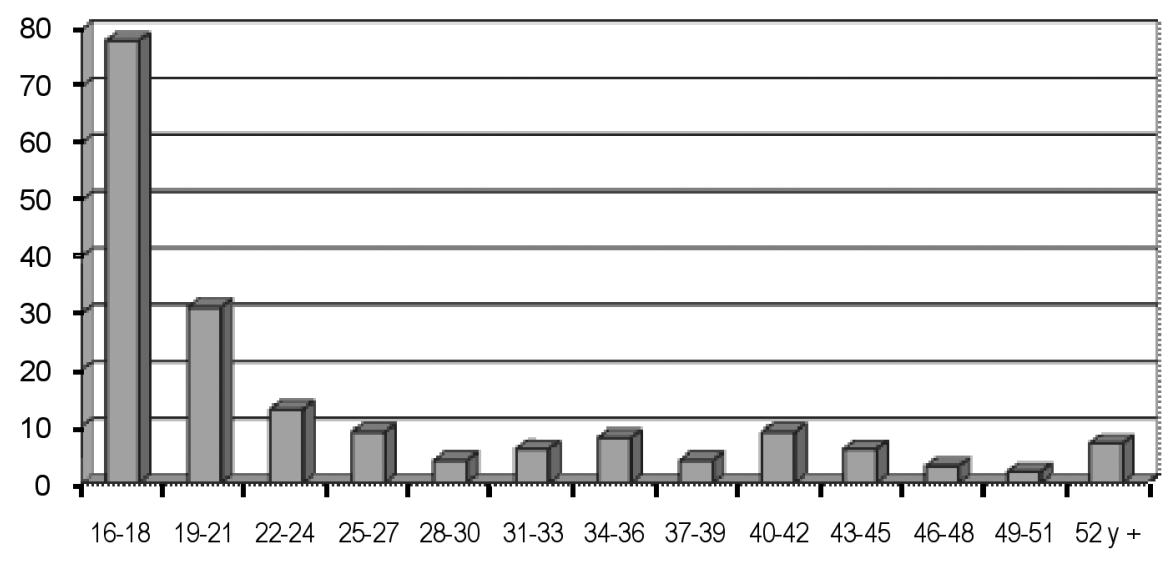

口 Sujetos por intervalo de edad 
entre los valores extremos de 16 hasta 53 años ambos incluido. La media de edad fue $24.5 \mathrm{y}$ la desviación típica, 12.14 .

En cuanto a la muestra de profesores, estuvo constituida por 12 personas- 7 mujeres y 5 hombresde los cuatro Centros de Adultos participantes, con una edad media estimada de unos cuarenta años, ya que nos fue imposible obtener la edad real.

\section{Materiales}

a) Se ha usado el Cuestionario de Clima Motivacional de Aula, CMC-Q (Alonso Tapia y Fernández Heredia, 2008). Este cuestionario evalúa el clima motivacional del aula en función del grado en que los profesores se caracterizan por usar las dieciséis pautas docentes que aparecen en la Tabla 1. Los ítems se contestan en una escala Likert de cinco puntos, mediante la que los alumnos señalan su grado de acuerdo con el contenido del ítem, y son de tipo positivo y negativo para evitar los efectos de la aquiescencia. Las características psicométricas del CMC-Q son satisfactorias -consistencia interna según las muestras .92 y .93 , habiéndose obtenido una validez muy adecuada tanto en los análisis confirmatorios, como de correlaciones y de regresión.

b) En el estudio original se construyeron expresamente una serie de Escalas de Validación del CMC-Q, escalas que también van a ser utilizadas en nuestro estudio. En concreto se añadieron ocho ítems que se agrupan en dos escalas, una de motivación y otra de satisfacción. Los ítems que integran la escala de motivación evalúan, respectivamente, el interés por la materia, la habilidad percibida, el esfuerzo y las expectativas de éxito. Correlacionaron de forma positiva y significativa con la puntuación en el CMC-Q pero, dada su redacción, pueden actuar como antecedentes o consecuentes del clima motivacional de clase percibido (ver Alonso Tapia y Fernández Heredia, 2008).

c) Con el propósito de evaluar si las orientaciones motivacionales influyen en la percepción del

Tabla 1. Pautas docentes evaluadas por el CMCQ y la escala-criterio, con ítems ejemplo

CMCQ Variables

El profesor usa situaciones novedosas. Este profesor presenta a menudo información nueva que hace que aumente nuestro interés.

El profesor evalúa los conocimientos precios. Este profesor explora lo que sabemos sobre un tema antes de explicarlo.

El profesor relaciona diferentes temas. Este profesor trata de ayudarnos a relacionar las nuevas ideas con lo que ya sabemos.

El profesor fomenta la participación. A este profesor le gusta que participemos, nos escucha y responde a nuestras preguntas.

Los mensajes de este profesor orientan al aprendizaje. A este profesor le gusta que disfrutemos aprendiendo cosas nuevas.

Los objetivos de aprendizaje están claros. (-) Este profesor cambia a cada momento y esto nos llena de confusión.

La actividad está bien organizada. En esta clase las instrucciones par alas tareas están clara por lo que sabemos qué es lo que tenemos que hacer.

El profesor promueve la autonomía. (-) Este profesor nunca nos da libertad para elegir cómo o con quién trabajar.

El profesor enseña a trabajar paso a paso. Este profesor explica paso a paso y eso hace que sea fácil entenderle.

El profesor usa muchos ejemplos. (-) Este profesor casi nunca pone ejemplos y eso hace que sea difícil entenderle.

El profesor lleva un ritmo adecuado. Este profesor se adapta a nuestro ritmo de aprendizaje dándonos tiempo para pensar.

El profesor proporciona retroalimentación que ayuda a aprender de los errores. Este profesor te hace sentir que puedes aprender de los errores.

El profesor evalúa "para" ayudar a aprender. (-) Este profesor te pone exámenes que tienen poco que ver con lo que se ha hecho al trabajar en clase.

El profesor elogia los progresos de los alumnos. Este profesor elogia nuestros esfuerzos por aprender siempre que tiene ocasión.

El profesor trata a los alumnos con equidad. (-) Este profesor presta más atención a los alumnos más inteligentes.

El profesor se preocupa por cada alumno. (-) hay pocos alumnos que hagan preguntas porque este profesor es muy distante y no suele ayudar.

Escala criterio

Satisfacción con el trabajo del profesor. Si se pudiese escoger profesor, aconsejaría escoger al mío. 
CMC, se aplicó a la muestra de estudiantes el Cuestionario de Evaluación de la Motivación en Sujetos Adultos, MAPE-3 (Alonso Tapia y Huertas, 2000), que permite definir dos dimensiones y unir dos tipos de orientaciones motivacionales (orientación al aprendizaje versus orientación al resultado). Las siete escalas de que consta son: miedo al fracaso, deseo de éxito y reconocimiento, motivación externa y ansiedad facilitadora del rendimiento, escalas que integran el factor "Orientación al resultado", y las escalas motivación por aprender, disposición al esfuerzo, y desinterés por el trabajo y rechazo del mismo, que integran el factor "Orientación al aprendizaje". Las características psicométricas del MAPE-3 son satisfactorias, con una consistencia interna media (alfa de Cronbach) de 0.79.

d) A los profesores, se les aplicó el Cuestionario de Expectativas y Actitudes Motivacionales del Profesorado, AMOP-B (Alonso Tapia, 1992), que incluye cuatro factores diferentes que intervienen en los patrones de enseñanza de los profesores. Se justifica la elección de este cuestionario porque, como ya hemos indicado, hay evidencia empírica de que los factores motivacionales de los profesores afectan al modo en que actúan en clase, e influyen en las percepciones de CMC por parte de sus alumnos. Si bien este influjo aún no ha sido comprobado aún en el caso de los adultos. Las escalas correspondientes a estos factores son: a) Escala de rechazo del trabajo en grupo y actitud pesimista respecto a la posibilidad de motivar; b) Escala de optimismo motivacional y de orientación al proceso; c) Actitud favorable al uso de la competición y la amenaza y orientación al resultado; y d) Escala de pesimismo generalizado.

\section{Procedimiento}

Se indicó a los estudiantes que debían responder al CMCQ en relación con lo que percibían en una asignatura concreta -Geografía e Historia; Ciencias de la Naturaleza; Lenguaje; Matemáticas; Enseñanza Globalizada (una adaptación curricular grupal no significativa de las asignaturas de Geografía e Historia, Ciencias de la Naturaleza, Lenguaje, Matemáticas e Inglés); e Inglés. A continuación, se les aplicó el MAPE-3 durante una sesión de tutoría de unos 50 minutos. Por su parte, a los profesores se les pidió que rellenasen el cuestionario AMOP-B.

\section{Análisis de datos}

Una vez recogida la información de los cuestionarios, se llevaron a cabo los siguientes análisis:

1) Con carácter piloto, debido al limitado tamaño de la muestra, se usaron técnicas de análisis confirmatorio del programa Amos para estimar el modelo base que hemos extraído de las consideraciones teóricas (AFC1). Se han obtenido las estimaciones usando el método de máxima verosimilitud. La valoración del ajuste del modelo se llevó a cabo usando índices de ajuste absolutos ( $\left.\mathrm{x}^{2}, \mathrm{x}^{2} / \mathrm{df}, \mathrm{GFI}\right)$, índices de ajuste incremental (IFI) e índices de ajuste no centralizados (CFI, RMSEA), y como criterio para la aceptación o el rechazo del grado de ajuste del modelo se utilizaron los niveles descritos por Hair y col (2006).

2) A continuación se realizó un estudio de validez cruzada de los resultados de nuestro estudio con el de Alonso Tapia y Fernández (2008) mediante análisis confirmatorio multigrupo (AFC2). Se usó la muestra de alumnos de Bachillerato del estudio original y nuestra muestra de adultos. El modelo inicial se utilizó como base para hacer las comparaciones sin restricción alguna de igualdad de parámetros entre las muestras. Posteriormente, se compararon otros dos modelos con el modelo inicial en los que se impusieron restricciones para diferentes conjuntos de parámetros: a) El modelo en el que se impone la igualdad de los 
pesos en la regresión y b) el modelo en el que se impone la restricción adicional de que las varianzas de los errores deben ser iguales. La evaluación del descenso relativo en bondad de ajuste se realizó por medio de la diferencia en el estadístico Ji- cuadrado entre los modelos con restricciones y el modelo sin restricciones.

3) En tercer lugar se calcularon las correlaciones entre el CMC y las variables motivacionales que pueden depender del mismo -interés, habilidad percibida, esfuerzo y expectativas de resultado- en combinación y por separado, y se realizaron cuatro análisis de regresión para determinar el grado en que el CMC y las variables mencionadas permiten predecir $-\mathrm{y}$ tal vez condicionar- la "satisfacción con el profesor" y el "rendimiento académico".

4) Para poder identificar qué factores personales de los alumnos adultos modulan la percepción del CMC, se analizaron las correlaciones entre las puntuaciones en las escalas de motivación y el CMC, y luego se realizó un análisis de regresión usando como criterio el cuestionario CMC-Q y como predictor el cuestionario MAPE-3.

5) Asimismo, para identificar las características del profesor que modulan el modo en que los alumnos adultos perciben el CMC se calcularon las correlaciones entre las puntuaciones de los profesores en el cuestionario AMOP y la puntuación media de los alumnos de su clase en el CMC y, posteriormente se realizó un análisis de regresión múltiple usando como criterio la variable "clima de aula" y como variables predictoras las características motivacionales de los profesores evaluadas mediante la prueba AMOP.

6) Finalmente, se realizaron una serie de ANOVAs de las puntuaciones en el CMC-Q utilizando como variable independiente el profesor y como variables dependientes la puntuación total y las puntuaciones en cada una de las variables componentes del mismo, para determinar si existen diferencias significativas -desde el punto de vista de los alumnos- en el clima creado por el profesorado.

\section{Resultados}

\section{Análisis Factorial Confirmatorio}

La Figura 2 muestra las estimaciones estandarizadas de los parámetros del modelo confirmatorio. Todos los pesos estimados $(\lambda)$ son significativos $(\mathrm{p}<0.001)$.

La Tabla 2 muestra los estadísticos de ajuste del modelo propuesto (AFC-1). El estadístico Ji- cuadrado es significativo debido probablemente al tamaño de la muestra, pero el cociente $\chi^{2} / g l$ así como los restantes índices se sitúan dentro de los límites que permiten la aceptación del modelo $\left(\chi^{2} / g l=1.90<5 ;\right.$ IFI (incremental fit index $)=.92>$ .90; CFI (comparative fit index) $=.92>.90 ; \mathrm{y}$ RMSEA (root mean square error of approximation) $=.05<.08$ ). Se exceptúa el GFI (goodness of fit index $)=.88<.90$, que queda justo al límite de la significación (ver tabla 2 ).

\section{Análisis de validación cruzada multi-grupo}

Para comprobar la validez cruzada se realizó un análisis multigrupo utilizando, además del grupo de adultos, el grupo de Bachillerato del estudio de Alonso Tapia y Fernández (2008). En relación con este análisis, los índices de ajuste caen dentro de límites aceptables (Tabla 2, AFC2), y los estadísticos de comparación del modelo que se presentan en la Tabla 3 (AFC2) muestran que el ajuste no se reduce de forma significativa incluso si se imponen restricciones sobre los pesos de la regresión pero sí, si se impone la igualdad de los residuos. Esto significa que hay algún aspecto en el que la valoración que adolescentes y adultos hacen de los patrones de actuación que definen el clima motivacional de clase difiere en algún aspecto. Por esta razón, para determinar qué pautas de actuación de los profesores son 
Figure 2. CMC-Q: AFC: Solución inicial estandarizada ${ }^{1}$

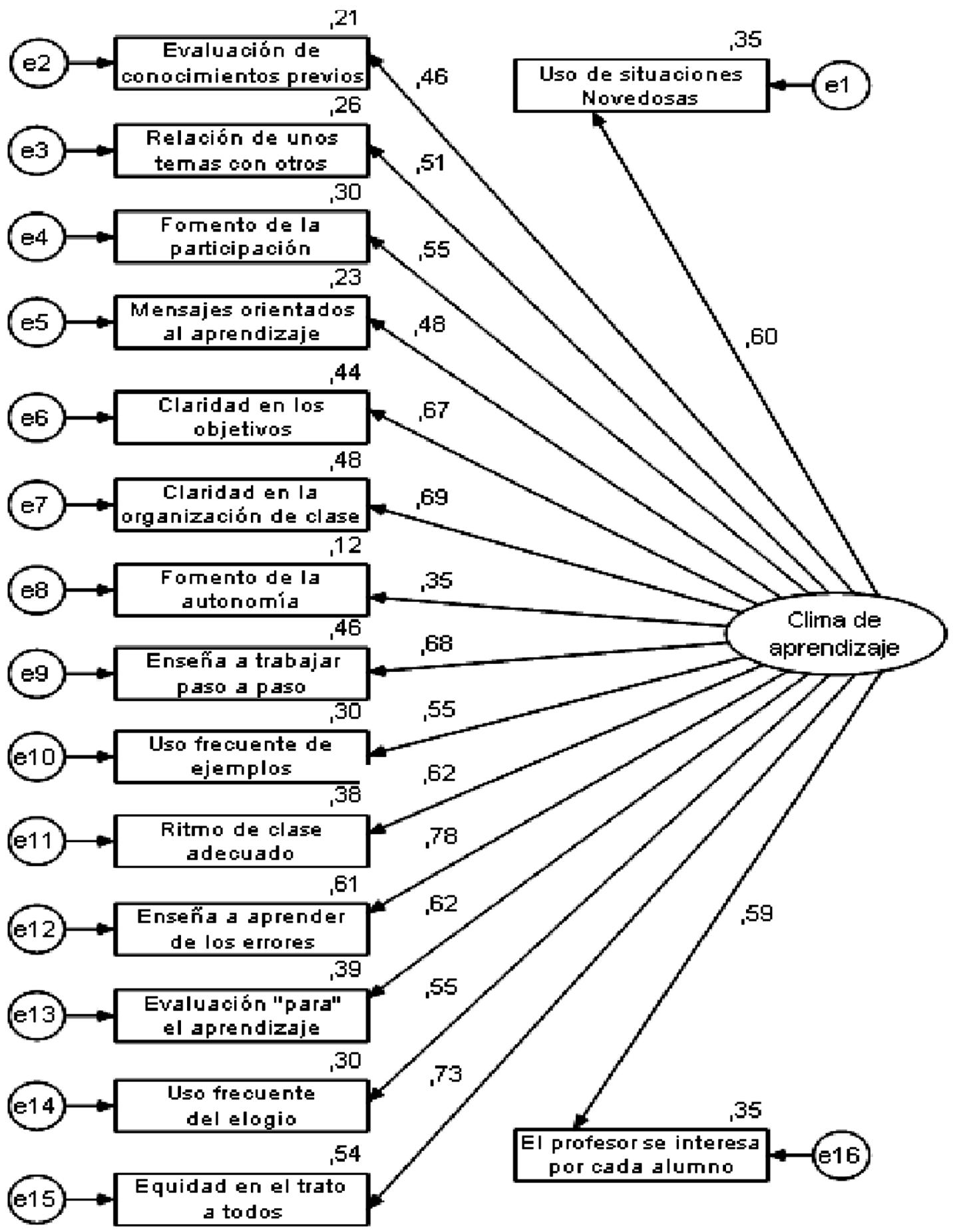

1 "e": error correspondiente a cada variable.

valoradas de modo diferente por los alumnos de uno y otro nivel -qué relaciones en el modelo diferían significativamente- se compararon los resultados relativos a cada relación utilizando el test z propues- to por Clogg, Petkova y Haritou (1995). Sólo se encontró una diferencia significativa entre dos coeficientes de regresión, y otra que roza el límite de significación. La primera diferencia tiene lugar en la 
Tabla 2. Índices de bondad de ajuste de los distintos análisis confirmatorios realizados

\begin{tabular}{lcccccccc}
\hline & $c h i^{2}$ & $D f$ & $P$ & chi ${ }^{2} / d f$ & GFI & IFI & CFI & RMSEA \\
\hline AFC 1 & 400.118 & 210 & .000 & 1.905 & .883 & .924 & .924 \\
AFC 2 & 168.95 & 31 & .000 & 5.45 & .842 & .868 & .868 & .050 \\
\hline
\end{tabular}

valoración que los alumnos hacen de la claridad de organización de la clase, claridad que se asocia a un buen clima motivacional más en los adolescentes de Bachillerato que en los adultos (Diferencia $=-0.37$; $\mathrm{z}=-2.71)$. En cuanto a la segunda, aparece en la valoración de la retroalimentación que permite aprender de los errores (Diferencia: 0.27; $z=1.82$ ), diferencia que implica que el hecho de que el profesor ayude a aprender de los errores es considerado por los adultos más que por los alumnos de Bachillerato como indicador de un buen clima motivacional. individuales de aprendizaje, incrementaría la capacidad del estudiante tanto de verse capacitado como de anticipar consecuencias positivas del propio desempeño y le despertaría un mayor interés por la asignatura.

En segundo lugar cabe señalar la elevada correlación positiva y significativa entre el CMC y la "Satisfacción" con el trabajo del profesor, resultado que concuerda con nuestra hipótesis, y la correlación positiva y significativa, aunque no muy alta, entre el CMC y el rendimiento evaluado a partir de las notas.

Tabla 3. Validación cruzada del modelo a través de análisis multigrupo en el que se comparan dos muestras. Diferencias en Ji-cuadrado al comparar los distintos modelos con el modelo sin restricciones

\begin{tabular}{|c|c|c|c|c|}
\hline Análisis & Modelo & $\mathrm{DF}$ & Ji- cuadrado & $\mathrm{P}$ \\
\hline ACF2: Bachillerato-Adultos & $\begin{array}{l}\text { Pesos de la regresión } \\
\text { Residuos }\end{array}$ & $\begin{array}{l}15 \\
31\end{array}$ & $\begin{array}{r}18.07 \\
168.95\end{array}$ & $\begin{array}{l}.259 \\
.000\end{array}$ \\
\hline
\end{tabular}

\section{Análisis de correlaciones entre CMC y variables} que pueden depender del clima de clase

La Tabla 4 muestra las correlaciones entre el CMCQ, las variables motivacionales por separado, las escalas de "motivación" y satisfacción con el trabajo del profesor" y el rendimiento. Cabe destacar, en primer lugar, que el Clima Motivacional de Clase correlaciona positivamente y de modo significativo, como se esperaba, con todas las variables motivacionales a las que podría afectar -"Habilidad Percibida", "Expectativas de resultado", "Esfuerzo por aprender" e "Interés por la asignatura"-, tanto si se consideran por separado como en conjunto. Además, las correlaciones entre las mismas justifican que puedan agruparse en una medida única de motivación. Esta relación sugiere, aunque no se pueda concluir por tratarse de datos correlacionales que sólo reflejan "asociación", que un clima de aprendizaje podría influir en el desarrollo de metas

\section{Análisis de regresión en los que el CMC se utiliza como variable predictora.}

Teniendo en cuenta las correlaciones anteriores, se han realizado cuatro análisis de regresión utilizando el método "hacia atrás" para ver en qué grado el clima motivacional del aula y la motivación -medida de forma global- o las características motivacionales específicas potencialmente sensibles al influjo del entorno -interés, habilidad percibida, esfuerzo y expectativas de éxito- contribuyen a predecir la satisfacción del alumno (Análisis 1 y 2) y el rendimiento (Análisis 3 y 4). Los resultados se presentan en la Tabla 5. Como puede verse, la satisfacción del alumno se ve influida principalmente por el clima de clase, aunque la motivación evaluada globalmente también tiene un peso significativo. Sin embargo, cuando la medida global se sustituye por la de los cuatro componentes, sólo lo habilidad percibida contribuye de modo significativo, junto con 
Tabla 4. Correlaciones entre las variables que pueden depender del clima de clase

\begin{tabular}{|c|c|c|c|c|c|c|c|}
\hline $\mathrm{N}: 168$ & Interés & $\begin{array}{l}\text { Habilidad } \\
\text { percibida }\end{array}$ & $\begin{array}{l}\text { Expectativas } \\
\text { de resultado }\end{array}$ & Esfuerzo & Motivación & Satisfacción & $\begin{array}{r}\text { NOTAS } \\
\mathrm{N}: 144\end{array}$ \\
\hline $\mathrm{CMC}$ & $.413^{* *}$ & $.391 * *$ & $.509 * *$ & $.353 * *$ & $.604 * *$ & $.733 * *$ & $.272 * *$ \\
\hline Interés & & $.413 * *$ & $.290 * *$ & $.223^{* *}$ & $.687 * *$ & $.387 * *$ & $.191 *$ \\
\hline Habilidad percibida & & & $.179 *$ & $.304 * *$ & $.673 * *$ & $.378 * *$ & $.211^{*}$ \\
\hline Expectativas de resultado & & & & $.390 * *$ & $.682 * *$ & $.429 * *$ & $.227 * *$ \\
\hline Esfuerzo & & & & & $.713 * *$ & $.307 * *$ & .029 \\
\hline Motivación & & & & & & $.543 * *$ & $.240 * *$ \\
\hline Satisfacción & & & & & & & $.224 * *$ \\
\hline
\end{tabular}

* La correlación es significativa al nivel 0,05 (bilateral).

el clima de clase, a la satisfacción del alumno. Así mismo puede verse que en el caso del rendimiento, el clima de clase también tiene un peso significativo, pero no así las variables motivacionales evaluadas, ya se tomen en conjunto o por separado. caso de la disposición al esfuerzo la relación es nula, hecho que parece implicar que la percepción de que el clima de clase está orientado al aprendizaje no depende del grado en que está dispuesto a esforzarse el alumno, sino de otros factores. En conjunto

Tabla 5. Análisis de regresión

\begin{tabular}{|c|c|c|c|c|c|c|c|c|}
\hline \multirow[b]{2}{*}{ Criterio } & \multirow[b]{2}{*}{$\mathrm{R}^{2}$} & \multirow[b]{2}{*}{$\mathrm{P}$} & \multicolumn{6}{|c|}{ Predictores: Coeficientes de regresión estandarizados } \\
\hline & & & $\begin{array}{l}\text { Clima de } \\
\text { aprendizaje } \\
\text { (CMC) }\end{array}$ & Motivación & $\begin{array}{l}\text { Habilidad } \\
\text { Interés }\end{array}$ & percibida & Esfuerzo & $\begin{array}{c}\text { Expectativa } \\
\text { de éxito }\end{array}$ \\
\hline Satisfacción & .552 & .000 & $.637 * * *$ & $.159 * *$ & & & & \\
\hline Satisfacción & .541 & .000 & $.691 * * *$ & & - & $.108^{*}$ & - & - \\
\hline Rendimiento & .064 & .001 & $.272 * *$ & - & & & & \\
\hline Rendimiento & .064 & .001 & $.272 * *$ & & - & - & - & - \\
\hline
\end{tabular}

$1 * * * \mathrm{p}<.001 ; * * \mathrm{p}<.01 ; * \mathrm{p}<.05$.

\section{Análisis de correlaciones entre las variables de los alumnos que pueden modular la percepción del CMC, evaluadas mediante el MAPE-3, y el CMC-Q}

Según los resultados recogidos en la Tabla 6 muestran, por un lado, que existe una correlación negativa y significativa entre la Orientación al Resultado y el CMC, correlación así mismo negativa con todos los factores específicos que integran esta orientación, si bien sólo en dos casos llega a ser significativa. Y, por otro lado, que la Orientación al Aprendizaje correlaciona positiva y significativamente con el CMC, si bien en este caso sólo con dos de los tres componentes de esta orientación sigue siendo significativa la correlación. Tan sólo en el estos resultados son los que cabía esperar, dado el significado de los factores evaluados (Alonso Tapia, Montero y Huertas, 2000).

\section{Análisis de regresión en los que las motivaciones de los alumnos son las variables predictoras y el CMC es la variable criterio}

Con el fin de determinar en qué medida cada una las características motivacionales con que los sujetos adultos acceden a clase añade algo a la modulación de la percepción del CMC, se han hecho dos análisis de regresión utilizando el método "hacia atrás" en los que se han utilizado como predictores, respectivamente, las puntuaciones en los factores de 
Tabla 6. Correlaciones entre los factores de primer y segundo orden del MAPE, y el CMC

\begin{tabular}{ll}
\hline MAPE-3 & CMC \\
\hline Orientación al resultado (OR) & $-.213^{* *}$ \\
Miedo al fracaso (MFR) & -.091 \\
Deseo del éxito y su reconocimiento (DER) & $-.233^{* *}$ \\
Motivación externa (MEX) & $-.221^{* *}$ \\
Ansiedad facilitadora del rendimiento (AFR) & -.133 \\
Orientación al aprendizaje (OA) & $.320^{* *}$ \\
Motivación por aprender (MAP) & $.442^{* *}$ \\
Disposición al esfuerzo (DES) & -.051 \\
Evitación del trabajo y rechazo de la tarea (-) (ERT) & $-.249^{* *}$ \\
\hline * La correlación es significativa al nivel 0.05 (bilateral). & \\
\hline
\end{tabular}

primer y segundo orden del MAPE 3. Los resultados se presentan en la Tabla 7. de forma significativa a tres de las variables en las que se ha evaluado a los profesores. Primero, cuanto más rechaza el profesor el trabajo en grupo (RTG) -y cuanto menos lo utiliza, por tanto-, los alumnos perciben que el clima de clase está más orientado al aprendizaje. Segundo, y contra lo que se esperaba, cuanto mayor es el optimismo motivacional del profesor y su orientación al proceso (OPT), menor es el grado en que los alumnos consideran que el CMC está orientado al aprendizaje. Se trata de resultados un tanto desconcertantes sobre los que volveremos en las conclusiones. Y tercero, cuanto mayor es el uso de la competición, menor es la percepción de que el CMC está orientado al aprendizaje.

Tabla 7. Análisis de regresión. Criterio: Satisfacción con el trabajo del profesor.

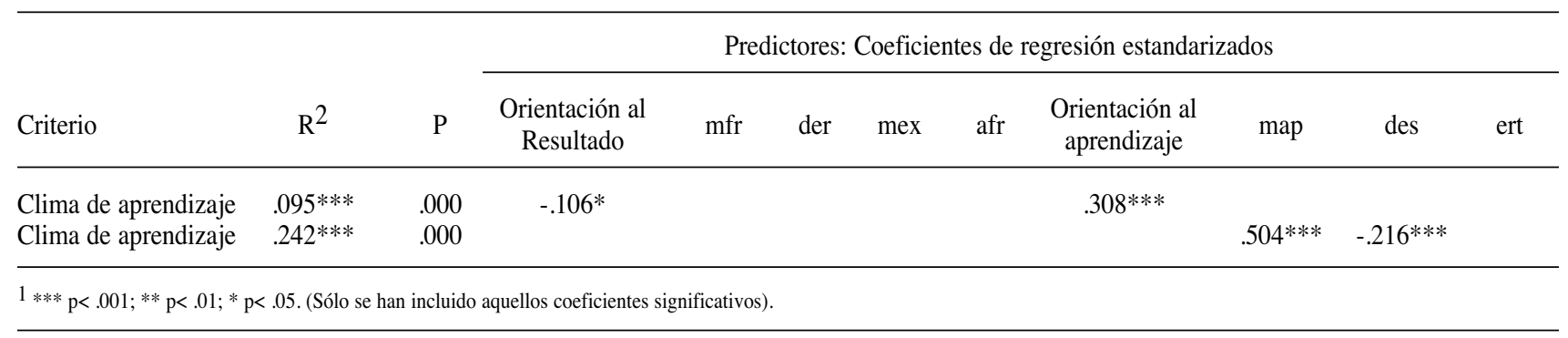

Como puede comprobarse, cuando se utilizan como predictores las orientaciones motivacionales, a medida que aumenta la orientación al aprendizaje, la percepción del CMC como orientado al aprendizaje tiende a aumentar, al contrario de lo que ocurre con la orientación al resultado, si bien en este caso el resultado apenas llega a ser significativo. Además, si se utilizan como predictores las motivaciones específicas, en este caso es el deseo de aprender el que se asocia con la percepción positiva del clima motivacional, mientras que la disposición al esfuerzo lo hace de forma negativa.

\section{Análisis de correlaciones entre las variables de los profesores que pueden modular la percepción del CMC evaluadas mediante el cuestionario AMOP- B y el CMC- $Q$}

Según los datos de la Tabla 8, el CMC se asocia
Análisis de regresión en el que las motivaciones de los profesores son las variables predictoras y el CMC es la variable criterio

Con el fin de determinar en qué medida cada una las características de los profesores añade algo a la modulación de la percepción del CMC, dado que existen correlaciones entre las mismas, se ha hecho un análisis de regresión utilizando el método "hacia atrás" en el que se han utilizado como predictores las puntuaciones en las cuatro escalas del cuestionario AMOP-B. Los resultados se presentan en la Tabla 9. Como puede verse, sólo dos de las variables que correlacionaban de forma significativa con el CMC contribuyen a explicar la varianza en el CMC de modo significativo, el "Rechazo de trabajo en grupo" que contribuye de forma positiva y el "Uso de la competición", que lo hace de forma negativa. 
Tabla 8. Correlaciones entre las variables del profesor que pueden afectar al Clima de Clase y el CMC

\begin{tabular}{lcccc}
\hline N: 168 & $\begin{array}{c}\text { Rechazo del trabajo } \\
\text { en grupo (RTG) }\end{array}$ & $\begin{array}{c}\text { Optimismo } \\
\text { motivacional (OPT) }\end{array}$ & $\begin{array}{c}\text { Pesimismo } \\
\text { generalizado (PESG) }\end{array}$ & $\begin{array}{c}\text { Uso de la competición } \\
\text { (UCO) }\end{array}$ \\
\hline Clima Motivacional de Clase (CMC) & $.315^{* *}$ & $-.261^{* *}$ &,- 066 & $-.259^{* *}$ \\
\hline$* *$ La correlación es significativa al nivel 0,01 (bilateral). & & & \\
\hline
\end{tabular}

Tabla 9. Análisis de regresión

\begin{tabular}{|c|c|c|c|c|c|c|}
\hline \multirow[b]{2}{*}{ Criterio } & \multirow[b]{2}{*}{$\mathrm{R}^{2}$} & \multirow[b]{2}{*}{$\mathrm{P}$} & \multicolumn{3}{|c|}{ Predictores: Coeficientes de regresión estandarizados } & \multirow[b]{2}{*}{$\begin{array}{l}\text { Uso de la } \\
\text { competición } \\
\text { (COMP) }\end{array}$} \\
\hline & & & $\begin{array}{c}\text { Rechazo del } \\
\text { trabajo en grupo } \\
\text { (RTG) }\end{array}$ & $\begin{array}{l}\text { Optimismo } \\
\text { Motivacional } \\
\text { (OPT) }\end{array}$ & $\begin{array}{l}\text { Pesimismo } \\
\text { generalizado } \\
\text { (PESG) }\end{array}$ & \\
\hline $\begin{array}{l}\text { Clima de aprendizaje } \\
\text { (CMC) }\end{array}$ & $.149 * * *$ & .000 & $.289 * * *$ & No Significativo & No Significativo & $-.226 * * *$ \\
\hline
\end{tabular}

\section{ANOVA de las diferencias en CMC entre profesores}

Al igual que se hizo con la muestra de bachillerato, el análisis de las puntuaciones de CMC de los 12 profesores, puntuaciones que se presentan en la Figura 3, puso de manifiesto la existencia de diferencias significativas $\left(\mathrm{F}_{\mathrm{gl}}: 11,156=3.72, \mathrm{p}<\right.$ .0001). Estas diferencias se encontraron así mismo no sólo en la puntuación total, sino también en la casi totalidad de las variables que integran el CMC$\mathrm{Q}$, aunque no se presentan estos datos por razón de espacio.

\section{Discusión e implicaciones para la enseñanza y la investigación}

El planteamiento de este trabajo ha sido desde el principio, el poder ofrecer a los profesores de estu-

Figura 3. Diferencias entre profesores en el grado en que el CMC es percibido como orientado al aprendizaje

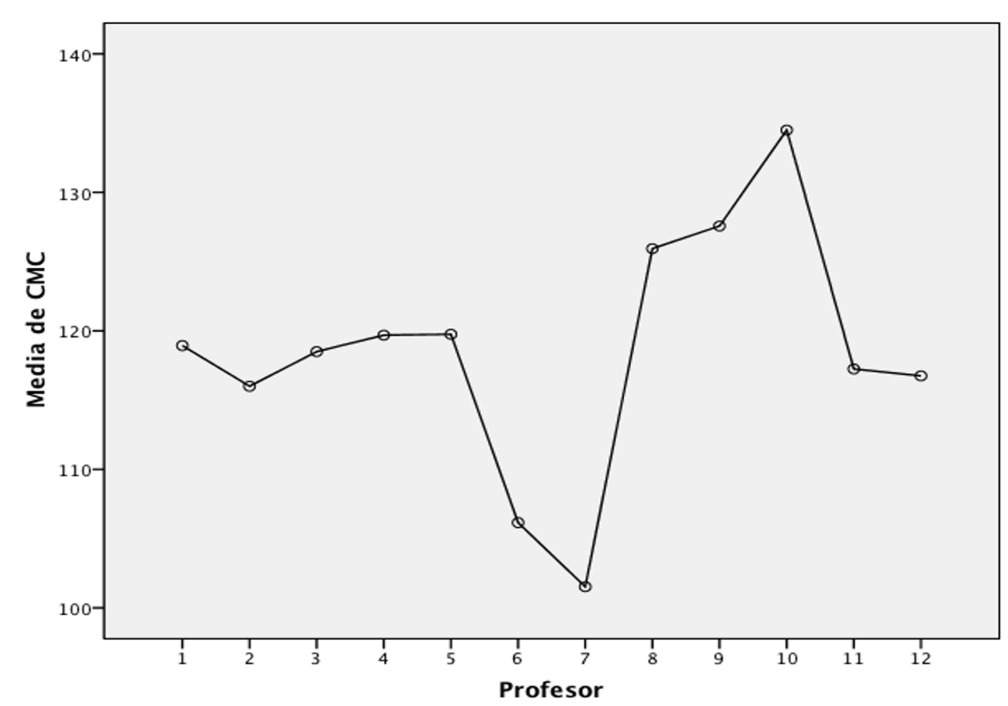


diantes adultos no universitarios, orientaciones sobre cómo motivar a sus alumnos en su asignatura. La idea apareció al estudiar los resultados ofrecidos por Alonso Tapia y Fernández Heredia (2008) relativos a los estudiantes adolescentes. De hecho, una de las cuestiones cruciales ha sido plantearse si podíamos aportar evidencia de que los resultados que obtuviéramos con los estudiantes adultos no universitarios, iban a ser similares a los conseguidos con los estudiantes adolescentes.

$\mathrm{Y}$ afortunadamente, los resultados que obtuvimos, a nivel general, han sido los esperados. De manera que se puede concluir que el clima motivacional de aula funciona de manera similar en las clases con estudiantes adultos no universitarios que con estudiantes adolescentes. Así que ¿qué características ha de reunir un profesor que quiera que sus estudiantes puedan percibir un CMC de aprendizaje en su asignatura? Las que se recogen en la Figura 2 , que son las que definen un clima motivacional orientado al aprendizaje, a saber: introducir las clases planteando problemas novedosos, explorar lo que el alumnado sabe sobre los contenidos para partir de estos conocimientos al explicar, ayudarle a relacionar temas y conocimientos dispersos, fomentar la participación escuchando y respondiendo a los alumnos de modo constructivo, señalar que lo importante es aprender, establecer los objetivos de aprendizaje de forma precisa, organizar las actividades de forma clara de modo que los alumnos no se pierdan, explicar los contenidos y enseñar a trabajar por pasos, usar muchos ejemplos, adaptarse al ritmo de aprendizaje y dar tiempo para pensar, proporcionar retroalimentación frecuente y precisa, evaluar de forma que se vea que el objetivo es ayudar a aprender, elogiar los progresos de los alumnos, tratarles con equidad y mostrarse cercano a ellos, tratando de ayudarles. Si un profesor logra esta percepción en el estudiante, hará que aumenten su interés por aprender, el grado en que se siente capaz de lograrlo (habilidad percibida), el grado en que se esforzará por conseguirlo y las expectativas de conseguir el éxito.

Sin embargo, también se han encontrado algunas diferencias entre el valor que adolescentes y adultos no universitarios atribuyen a distintos aspectos de la actuación del profesor a la hora de considerar en qué medida el clima motivacional de clase se orienta al aprendizaje, diferencias que se hace necesario comentar. Y que indican la línea que deben tomar investigaciones futuras:

En primer lugar, aunque los resultados obtenidos en ambos tipos de estudiantes sean similares, hay que hacer dos importantes matices: por un lado, los alumnos adolescentes dan más importancia que los adultos al hecho de que la organización de la clase sea clara. Y por otro lado, los adultos dan más importancia que los adolescentes al hecho de que el profesor les ayude a aprender de sus propios errores. Puesto que, en cualquier caso la claridad es buena para el aprendizaje, aunque los adultos den menos importancia a este factor, será necesario seguir insistiendo a los profesores en la necesidad de la misma. Sin embargo, lo que parece especialmente importante es atender al mayor valor que dan los alumnos a aprender de los errores. Si los errores no se transforman en una ocasión para aprender, los adultos, muchos de los cuales tienen una historia de fracasos, probablemente verán las clases como una situación que pone de manifiesto sus limitaciones y que no les ayuda a superarlas y se desmotivarán.

En segundo lugar, parece ser que no solo los adultos se asemejan a los adolescentes en el valor que atribuyen a los distintos factores que configuran el clima motivacional de clase, sino también en cómo éste clima les afecta. Así, si un profesor logra construir un clima motivacional de aprendizaje, tanto los alumnos adultos como los adolescentes tienen más probabilidades de interesarse por las asignaturas, de valorar positivamente su capacidad por aprender, de desarrollar metas individuales de aprendizaje y esforzarse por lograrlas y de esperar conseguir el éxito final.

En tercer lugar, también hemos considerado relevante comprobar cuál es la relación que hay entre el tipo de clima motivacional que ha sido capaz de crear un profesor en su clase y la satisfacción que pueda tener un estudiante adulto con la asignatura 
que imparte porque en función de los resultados que obtuviéramos en esta cuestión, podríamos ofrecer a los profesores orientaciones sobre cómo motivar a futuros alumnos a escoger sus asignaturas y/o motivarles a que permanecieran con agrado en ellas. También en este apartado, nos preguntamos en qué medida influía un clima de clase percibido como satisfactorio en las notas obtenidas por el alumno adulto en cuestión. Y los resultados han sido curiosos: hemos visto que, en efecto y tal y como se esperaba, un clima de clase percibido como facilitador del aprendizaje, lograba que los adultos se sintieran muy satisfechos con el trabajo del profesor hacia ellos, pero no ha ocurrido lo mismo con el rendimiento o, mejor dicho, ha ocurrido lo mismo pero en mucho menor grado. Es decir, las calificaciones académicas obtenidas por los estudiantes adultos en las clases donde esos mismos estudiantes han percibido que el ambiente les motivaba a aprender, no se asocian a la percepción del clima de clase en el grado en que cabía esperar. Hay que indicar en este punto, que no quiere decir que el clima de clase no prediga unas buenas calificaciones académicas, sino que la predicción no es tan alta como se esperaba. Una posible interpretación de este hecho, podría ser que el esfuerzo invertido en un proceso de aprendizaje empieza a dar sus frutos a más largo plazo de lo que ha durado el tiempo de recogida de datos. En futuras investigaciones, podría ser posible hacer la recogida de datos en varios momentos posteriores, y así comprobar si la relación entre el clima motivacional y el rendimiento es tan alta como se esperaba.

En cuarto lugar, dado que algunos profesores habían comentado que por más estrategias docentes motivadoras que ponían en práctica dentro de sus clases, no veían la manera de "llegar" a determinados adultos, nos preguntamos en qué medida la meta individual de aprendizaje con que entraba un estudiante adulto a una clase, hacía como de filtro que pudiera tener una gran influencia en su percepción individual del clima motivacional real de la clase. Y fue este el motivo de aplicar el MAPE-3 a nuestra muestra de adultos y de comparar los resultados con los del CMC-Q. Esperábamos en este punto, que todo saliera según las suposiciones expuestas en el estudio de Alonso Tapia y Fernández Heredia (2008), esto es, que cuanto mayor fuese en los alumnos su orientación al aprendizaje, más valorarían como elementos constitutivos de un clima motivacional de aprendizaje las pautas de actuación recogidas en el cuestionario. Sin embargo, aunque los datos han confirmado nuestras suposiciones en su mayor parte, de nuevo hay que hacer dos importantes matizaciones. Por un lado, aunque los datos apenas alcanzan los valores estándar de significación, cuanto mayor es la orientación de los adultos al resultado, más negativamente han valorado el hecho de que los profesores actúen de acuerdo con las pautas que recoge el cuestionario, resultado paralelo al encontrado con adolescentes por Alonso Tapia y Pardo (2006). No está claro a qué puede deberse este hecho, pero la segunda matización quizás nos dé una pista. Por otro lado, si bien cuanto mayor es la orientación al aprendizaje, más positivamente se perciben las pautas de actuación incluidas en el cuestionario CMC-Q, uno de los factores que está incluido en la orientación individual de aprendizaje -la disposición al esfuerzo-, no se relaciona de ninguna manera con la percepción del clima motivacional de clase. Es decir, la disposición del estudiante adulto a esforzarse no parece asociada en la percepción que ha tenido del clima motivacional de su clase como clima de aprendizaje. Este resultado nos llamó la atención, porque estudios anteriores habían puesto de manifiesto que esta disposición es uno de los componentes fundamentales de la orientación al aprendizaje (Alonso Tapia, 2005a). Y la sorpresa fue mayor cuando en el análisis de regresión que realizamos después entre los resultados del MAPE-3 y el CMC$\mathrm{Q}$, la disposición al esfuerzo predice de forma negativa pero significativa -si bien en un grado muy pequeño- la valoración que los alumnos hacen de las pautas de actuación recogidas en el cuestionario como indicadoras de un clima motivacional orientado al aprendizaje. Parece, pues, que el esfuerzo sí que se asocia a la percepción del clima motivacional de clase, ya que parecía, que al fin y al cabo, el esfuerzo sí que parecía influir en la percepción del 
clima motivacional, aunque no en la línea esperada. Una posible interpretación de este resultado es que, a nivel práctico, aunque los adultos orientados al aprendizaje estén dispuestos a realizar el esfuerzo necesario para lograr sus objetivos académicos, no quieran asumir excesivos esfuerzos porque la valoración personal costo-resultado les sea desfavorable.

En quinto lugar, dado que en trabajos anteriores se ha comprobado que los profesores difieren en el clima motivacional que crean con sus pautas de actuación, hemos intentado ver a qué características de los mismos se deben estas diferencias. Partíamos del supuesto de que los profesores actúan sobre la base de sus conocimientos, expectativas y motivaciones, por lo que evaluamos sus motivaciones y expectativas respecto a su labor docente mediante el cuestionario AMOP-B y las pusimos en relación con las puntuaciones en el CMC-Q. Los resultados en parte han sido coherentes con lo esperado y en parte no. En primer lugar y en contra de lo esperado, cuanto mayor es el grado en que un profesor rechaza trabajar en grupo -y probablemente cuanto menos usa esta forma de trabajo-, más se considera que, en conjunto, su forma de actuación está orientada al aprendizaje y viceversa. Es posible que los condicionamientos socio-económicos de un adulto, acostumbrado a organizar su tiempo de una manera muy personal, le dificulten poder adoptar la flexibilidad necesaria como para poder juntarse con otros compañeros fuera del espacio de clase para realizar trabajos en grupo; o bien, existe una falta de seguridad en el adulto de lo que sabe y esto le haga rechazar el involucrarse en un grupo donde pudiera quedar en evidencia su ignorancia; o bien por último, haya una carencia de estrategias de trabajo relativas a la posibilidad de adaptación a un trabajo de tipo grupal, por la presencia de hábitos ya muy marcados de trabajo individual. Así mismo en contra de lo esperado, cuanto mayor es el optimismo del profesor respecto a la posibilidad de motivar y más trata de usar estrategias en este sentido, menor es el grado en que los alumnos adultos perciben su clase como orientada al aprendizaje. Aunque no tenemos una hipótesis clara para explicar este resultado, es posi- ble que un exceso de intención de motivar por parte del profesor, produzca rechazo en los adultos, porque esto podría implicar que tuvieran que invertir un esfuerzo mayor del que en principio, quisieran aportar a sus objetivos académicos. Esta explicación, por otra parte, es coherente con el papel negativo que el esfuerzo a realizar parece producir en la percepción que los adultos tienen del clima motivacional del aula.

En sexto y último lugar, queríamos simplemente comprobar si el cuestionario era suficientemente sensible para poner de manifiesto la existencia de diferencias en el clima motivacional creado por los profesores, comprobación que ha resultado afirmativa al igual que había ocurrido en el estudio de Alonso Tapia y Blanca con adolescentes. De hecho, el gráfico resultante de nuestro ANOVA, indica que algunos de los profesores puntúan muy por debajo de la media, por lo que requerían asesoramiento individualizado. Este hecho muestra que la evaluación del CMC mediante el CMCQ puede servir de base para ayudar a los profesores a tomar conciencia de las pautas de actuación docente que convendría modificar.

A modo de conclusión, podemos decir que a nivel general, los resultados de este estudio piloto, coinciden con los del trabajo de Alonso Tapia y Fernández (2008), por lo que cabe hacer a los profesores sugerencias de actuación análogas a las que se hacían allí. No obstante, dado el reducido tamaño de la muestra y dado que han aparecido algunos resultados inesperados, parece necesario ampliar aquella y reanalizar los datos, así como realizar un análisis jerárquico multinivel que permita determinar el peso relativo que las características de los profesores y las de los alumnos en la determinación de los resultados del CMC-Q. Y, finalmente, parece necesario intentar aclarar el papel que juega la disposición al esfuerzo en el estudiante adulto con respecto a su percepción del clima de aula y, a través de ésta, en el rendimiento. Por otra parte, dado el escaso número de estudios sobre motivación, autorregulación y aprendizaje realizados con alumnos adultos no universitarios parece necesario seguir desarrollando estudios de 
evaluación e intervención destinados a proporcionar fundamentos sólidos a la actividad educativa realizada con este alumnado.

\section{Referencias}

Alonso Tapia, J. (1991). Motivación y aprendizaje en el aula. Cómo enseñar a pensar. Madrid: Santillana.

Alonso Tapia, J. (1992a). ¿Qué es lo mejor para motivar a mis alumnos? Análisis de lo que los profesores saben, creen y hacen al respecto. Madrid: Servicio de Publicaciones de la Universidad Autónoma.

Alonso Tapia, J. (1992b). Motivar en la adolescencia. Teoría, evaluación e intervención. Madrid: Servicio de Publicaciones de la Universidad Autónoma.

Alonso Tapia, J. (1997). Motivar para el aprendizaje. Teoría y estrategias. Barcelona: EDEBÉ.

Alonso Tapia, J. y López, G. (1999). Efectos motivacionales de las actividades docentes en función de las motivaciones de los alumnos, en Pozo y Monereo (Eds), El aprendizaje estratégico. Madrid: Santillana.

Alonso Tapia, J., Montero, I. y Huertas, J.A. (2000). Evaluación de la motivación en sujetos adultos. El cuestionario MAPE-3. Trabajo no publicado. Registro de Propiedad Intelectual: 91.618. Madrid.

Alonso Tapia, J. (2005). Motivación para el aprendizaje: la perspectiva de los alumnos. En Pérez Solís, M. (Ed). La orientación escolar en centros educativos (pp. 209.242). Madrid: Ministerio de Educación y Ciencia.

Alonso Tapia, J. (2005a). Motives, expectancies and value-interests related to learning: The MEVA questionnaire. Psicothema, 17, 404-411.

Alonso Tapia, J. (2005b). Motivar en la escuela. Motivar en la familia. Madrid: Morata.

Alonso Tapia, J. y Fernández Heredia, B. (2008). Development and initial validation of the Classroom Motivacional Climate Questionnaire
(CMCQ). Psicothema, 20, 883-895.

Alonso Tapia, J. y Fernández Heredia, B. (2009). Cuestionario de clima motivacional de clase: Un estudio de su validez transcultural. Infancia $y$ Aprendizaje, 32, 597-612.

Alonso Tapia, J., Huertas, J.A. y Ruiz, M. (2010). On the nature of motivational orientations: Implications of assessed goals and gender differences for motivational goal theory. Spanish Journal of Psychology, 13, 231-242.

Alonso Tapia, J. y Pardo, A. (2006). Motivar en el aula. Madrid: Universidad Autónoma de Madrid.

Ames, R. (1983). Teachers' attributions of their own teaching. En J. Levine y M. Wang (Eds). Teacher and student perception: implications for learning. Hillsdale, NJ: Lawrence Erlbaum.

Ames, C. y Archer, J. (1988). Achievement goals in the classroom. Students'learning strategies and motivation process. Journal of Educational Psychology, 80, 260-267.

Ames, C. (1990). Achievement goals and classroom structure. Developing a learning orientation. Presented at Annual Metting of the American Educational Research Association, Boston.

Ames, C. (1992). Clasrooms: goals, structures and student motivation. Journal of Educational Psychology, 84, 261-271.

Atkinson, J.W. (1964). An introduction to Motivation. Princeton, NJ: Van Nostrand.

Assor, A. y Kaplan, H. (2001). Mapping the domain of autonomy support. In A. Efklides, J. Kuhl \& R.M. Sorrentino (Eds.), Trends and prospects in motivational research. (pp. 101-120). The Netherlands: Kluwer.

Bandura, A. (1987). Pensamiento y acción: fundamentos sociales. Editorial: Martínez Roca.

Bandura, A. (1977). Social Learning Theory. New York: General Learning Press.

Bandura, A. (1997). Self-efficacy: The exercise of control. New York: Freeman.

Bobbitt, S. (2007). Young Children's Motivation to Read and Write: Development in Social Contexts. Cognition and instruction, 25, 219-270.

Brophy, J.E. (2005). Goal theorists should move on 
from performance goals. Educational Psychologist, 40, 167-176.

Covington, M. (2000). Goal theory, motivation and school achievement: an integrative review. Annual Review of Psychology, 51, 171-200.

Elliot, A.J. \& Covington, M. V. (2001). Approach and avoidance motivation. Educational Psychology Review, 13, 73-92.

Elliot, A. y Dweck, C.S. (2005). Handbook of competence and motivation. Nueva York: Guilford Press.

Good, T.L. \& Brophy, J.E. (2000). Looking into classrooms. New York: Longman.

Hidi, S. (2006). Interest: A unique motivational variable. Educational Research Review, 1, 69-82.

Kuhl, J. y Beckmann, J. (Eds.) (1985). Action control: From cognition to behavior. New York: Springer-Verlag.

Midgley, C., Maher, M.L., Hruda, L.Z., Anderman,
E., Anderman, L., Freeman, K.E., Gheen, M., Kaplan, A., Kumar, R., Middleton, M.J., Nelson, J., Roeser, R., \& Urdan, T.(2000). Manual for the patterns of Adaptive Learning Scales. University of Michigan.

Pardo, A. y Alonso-Tapia, J. (1990). Motivar en el aula. Madrid: Universidad Autónoma.

Renninger, K.A., Hidi, S. \& Krapp, A. (Eds). (1992). The role of interest in learning and development. Hillsdale, NJ: Erlbaum.

Self-Brown, S.R. y Mathews-II, S. (2003). Effects of classroom structure on student achievement goal orientation. Journal of Educational Research, 97, 106-111.

Urdan, T. y Turner, J.C. (2005). Competence Motivation in the Classroom. In Elliot, A. y Dweck, C. (2005). Handbook of competence and motivation (pp. 297-317). New York, Guilford Press.

Manuscrito recibido: 02/08/2010

Revisión recibida: 30/09/2010

Manuscrito aceptado: 04/10/2010 